\title{
Sobre las tensiones en los equipos de las revistas científicas universitarias
}

On the tensions in the teams of university scientific journals

\author{
Guillermo O. Quinteros \\ Universidad Nacional de La Plata. Facultad de Humanidades y Ciencias de la Educación. \\ Centro de Historia Argentina y Americana, Argentina \\ goscarquinteros@gmail.com
}

\section{RESUMEN:}

El presente escrito tiene un doble propósito, a saber: por un lado, ilustrar sobre una parte de lo que el autor -en su carácter de editor de revistas científicas- recibe a modo de crítica, comentario o interrogantes, ya sea de los lectores, como de otros actores que están involucrados en la realización de una revista. Por otro lado, se expone lo que piensa el autor sobre quiénes son los realizadores de una revista científica universitaria y los roles y competencias de los equipos que la hacen. El trasfondo de la cuestión son las tensiones generadas en los equipos editoriales, a partir de la introducción de nuevas tecnologías en las formas de trabajar y la incorporación de normas de edición emanadas de organismos de evaluación científica nacionales y extranjeros.

Palabras Clave: Revistas científicas, Universidad, Tensiones, Editores .

\begin{abstract}
:
This paper has a double purpose, namely: on the one hand, to illustrate a part of what the author -in his capacity as editor of scientific journals- receives as a criticism, commentary or questions, whether from the readers, as of other actors who are involved in the realization of a journal. On the other hand, it is exposed what the author thinks about who are the makers of a university scientific journal and the roles and competences of the teams that make it. The background of the issue is the tensions generated in the editorial teams, from the introduction of new technologies in the ways of working and the incorporation of editing standards emanating from national and foreign scientific evaluation bodies.
\end{abstract}

KEYWORDS: Scientific journals, University, Tensions, Editors.

Tal vez esté equivocado y ya no sea de utilidad pensar en estas cosas, pero por si encuentro un lector ávido de saber sobre su propia actividad como escribidor y, más ampliamente, como intelectual, dejo estas líneas, sin pretensión científica alguna. ${ }^{1}$ Tampoco es mi deseo entrar en disputas políticas de ningún ámbito, ni de la propia Universidad, en la cual he ocupado diferentes roles dentro del equipo editor de la revista Trabajos y Comunicaciones 2 da época y como director y editor de la revista Cuadernos de H Ideas. ${ }^{2}$ El propósito es solo reflexionar sobre algunos aspectos que, por diferentes razones, me parecen poco dialogados y debatidos. ${ }^{3}$ Últimamente me han hecho algunas preguntas referidas a las revistas que de algún modo - debo ser sincero- me sorprendieron. Por la misma razón me encontraron poco preparado para responderlas - como cuando estamos concentrados en un tema y nos desvían a otro que nunca pensamos que podía surgir. En este caso supongo que daba por sentado que el interrogante no existía, porque las respuestas estaban claras. ¡Error!Me disculpo, entonces, por dejar un vacío, por no hacer lo que siempre he propuesto - dialogar - y, en consecuencia, por no practicar la docencia.

Hace poco, un colega escribió una larga reivindicación del libro (o, mejor dicho, de la escritura de libros) porque la tendencia actual es escribir papers para publicar en revistas y no libros. Coincido con Barriera, dado que es evidente que los sistemas de calificación de los organismos científicos — para la Argentina, fundamentalmente el CONICET ${ }^{4}$ - privilegian la cantidad de artículos publicados en revistas científicas 
bien posicionadas en los sistemas de evaluación especializados, para puntuar mejor a quienes integran las instituciones de investigación científica (Barriera, 2018, pp. 12-15). Este es un fenómeno de larga data y que formaría parte de otro debate, sobre las políticas de investigación, los organismos científicos y la organización de la ciencia en la Argentina y el mundo. Por el momento, cabe señalar que la tendencia marcada por Barriera dio como resultado la paulatina (pero constante) importancia que adquirieron las revistas. En la vorágine de un trabajo colectivo incrementado - entre otras cosas - por el número creciente de colaboraciones que los autores envían para su evaluación, y la notable transformación que significó comenzar a publicar en formato digital, estimo que se nos ha pasado el hecho de reflexionar sobre el propio medio.

Actualmente las revistas califican dentro de los organismos especializados, están o no indizadas, siguen los criterios de evaluación de tales organismos, requieren de procedimientos técnicos específicos para poder llegar a los lectores on line - porque descuento que ya no hablamos de las ediciones en papel, que todavía existen, claro, pero si no están en la web es como si no existieran-; luego de haber triunfado con una política a la que suscribimos están "colgadas" en acceso abierto, se gestionan mediante la plataforma OJS, etc., etc., etc. (Abadal y Alcaraz, 2006; Rozemblum y Banzato, 2009). Quienes venimos de un largo camino iniciado con una Olivetti portátil (para ser más específico, la de mi colega y amigo Guillermo Banzato) hemos procesado, cada quien como pudo, todos los cambios y transformaciones técnicas operadas en los últimos 40 años y, como buenos híbridos, estamos dispuestos a seguir evolucionando, adecuándonos a la tecnología del futuro. Pero, y tal vez por lo anterior, nos olvidamos de las respuestas - malas o buenas- procedentes no solo de nuestros mayores, sino de las discusiones políticas y conceptuales que daban tanto el marco como el sentido a las actividades que se desarrollaban. Desde luego me refiero a cuando éramos muy jóvenes y hacíamos los primeros pasos hacia la integración de los espacios universitarios de los que comenzábamos a sentirnos parte.

He aquí la pregunta que me llevó a escribir estas líneas, más otras que escuché hace unos años atrás, y algunos comentarios. “¿De quiénes son las revistas? ¿Es una pregunta legítima o no? Porque si decimos que es de la Facultad o si es de la Universidad es una cosa...”. De este modo se podía responder a la idea de si una Unidad Académica podía pedirle —o no- a una revista la adecuación a una forma de organización editorial interna, organización que probablemente se pueda realizar sin problema, sin inconveniente ni objeción, porque parece algo sin importancia. Pero la pregunta adquiere relevancia sobre todo porque parece que ya se tiene la respuesta, y porque se vincula con otras preguntas y/o comentarios que en apariencia ya fueron resueltos, como por ejemplo en el que sigue: "Me parece que debemos preguntarnos qué es lo que se publica y qué debemos publicar en las revistas de la Universidad”. Ante estas manifestaciones solo podemos preguntar cuáles son sus respuestas, quiénes toman las decisiones. Por favor, para evitar todo malentendido debería explicarse con algún detalle y con argumentos racionales, de quiénes son — a juicio de cada uno- las revistas; a quiénes deben responder; a quién se deben llevar las facturas y los gastos generados; qué debemos publicar y sobre la base de qué criterios. Sería muy bueno que nos permitan saber lo que piensan al respecto con total claridad, para poder así tener interlocutores con quienes eventualmente discrepar o establecer acuerdos. ${ }^{5}$

He aquí mis respuestas como editor para dejar abierto un diálogo, sin pretender tener la verdad sobre el asunto. ${ }^{6}$ En primer lugar, las revistas son de quienes las organizaron. Parece muy elemental, pero si observamos cómo surgieron, inmediatamente nos damos cuenta de que tal revista se originó por el impulso dado por tal grupo, tal sector o tal equipo dentro de tal o cual Secretaría, Departamento, Facultad o equipo de investigación. En general, una pequeña reseña del origen y la trayectoria de la revista se puede encontrar dentro de la misma, o lo más usual, en el "Quiénes somos” se expresa la constitución del grupo que dinamizó la creación de la misma. Allí también se encuentran los propósitos de la publicación, con las áreas de vacancia que se intenta cubrir, con el alcance tanto en sus contenidos como en el horizonte al que se aspira. Se suele expresar el objetivo de lograr intercambios de conocimientos científicos externos y compartir saberes que puedan enriquecer el conocimiento producido en los ámbitos locales. Cuando nace una revista todo es aspiracional: se plantean metas ideales, y si se quiere, utopías. Con el tiempo, se puede evaluar cuánto se ha logrado y qué queda por hacer, porque en tanto se van produciendo cambios, los equipos editoriales también 
cambian y replantean sus objetivos. Desde ya que la creación de una revista admite múltiples maneras y la descripta es una que, además, puede o no darse en determinadas situaciones y lugares. No obstante, una primera respuesta a la pregunta ¿de quién es la revista? la encontramos dentro de la misma revista. En el caso de que no la hallemos, ¿qué puede pasar? Pues dudaremos de su existencia, de su regularidad, y los organismos encargados de evaluarla dejarán de tenerla dentro de sus indizaciones. Pero esto es otra cuestión.

En segundo lugar, podemos ver que algunas revistas manifiestan que son representativas de una institución; por ejemplo, el órgano de difusión de una Facultad determinada, o la revista de un Departamento en particular. En estos casos, suponemos que existe tanto la necesidad como la voluntad de la Unidad Académica de que se trate, de sostener un órgano permanente de divulgación científica, para lo cual tomará las medidas que considere pertinentes a fin de solventarla, reclutar a los miembros para gestionarla, garantizar los medios técnicos para lograr su llegada al público lector, y decidirá eventualmente de quién dependerá la responsabilidad de mantenerla activa y sostener su regularidad. Es posible que sea la misma Facultad la que quiera establecer una política editorial determinada, y para ello deberá expresar con toda claridad los alcances y todo lo que se estila en cualquiera de las revistas del campo. Nuevamente se planteará quién o quiénes deciden tales cuestiones, y se responderá que lo harán aquellos que se considere, en ese momento particular, que representan mejor a los impulsores de tal emprendimiento. Se me ocurre pensar - si llegáramos a partir desde cero- que el Consejo Directivo podría elaborar los alcances, los objetivos y, más ampliamente, la política de la revista. Claro que los Consejos Directivos cambian, como también podrán cambiar los primeros criterios, a menos que consideremos que no se puede mejorar o revisar lo malo que pudo normarse en una circunstancia específica. Pero todo ello forma parte de la dinámica interna de la institución y del equipo editorial de que se trate. En estos casos la respuesta es igualmente rápida, dado que la revista sería de la Facultad.

En tercer lugar se encuentran aquellas revistas que, por decirlo de alguna manera, no están conformes con las existentes, o disienten respecto de las tendencias expresadas en una institución, y que aun formando parte de la misma, desean representar un enfoque distinto. Pueden ser la expresión de un grupo de trabajo, de un equipo de investigación, o de una línea teórica — metodológica o de carácter temático y empírico- que reúne a personas de distintas unidades académicas e incluso de distintas universidades. En este caso, ¿de quiénes son estas revistas? Volvemos a la primera respuesta: de quienes las hicieron, de quienes nació la idea, de quienes las impulsaron; en cuyo caso serán ellos quienes también fijen los alcances, la política editorial, etc.

A todo esto, hablamos de revistas cientificas, razón por la cual deberán cumplir con una serie de requisitos para lograr ese estatuto. ${ }^{7}$ ¿De quién depende esa cuestión? Pues de quienes están interesados en alcanzar esa calificación, que, tal como venimos planteando, podrán ser los grupos referidos o la Facultad, según el caso. Luego las revistas (me refiero a las cuestiones técnicas) son evaluadas externamente a la Unidad Académica o a la Universidad en donde han sido alojadas. Pero debo enfatizar que se ha promovido que las revistas fueran adecuándose a las normas y criterios de evaluación de los organismos externos a la Universidad o Facultad, cosa que en gran medida viene haciéndose con éxito. Al mismo tiempo se nos ha planteado con suma claridad que de los criterios que se establecieron con anterioridad a la creación de un organismo como por ejemplo Núcleo Básico, prácticamente no sirve ninguno. ${ }^{8}$ Hemos aceptado casi todas las adecuaciones del caso y los logros son evidentes para la UNLP. Está muy claro que las revistas que no cumplen con los requisitos de un organismo como el citado se caen de la indización y se terminó la historia. Si una Unidad Académica desea sostener una publicación que no mantiene su regularidad, hará lo que considere pertinente para preservarla o la dejará caer. En cambio, si no mantiene la regularidad y es una revista pensada como representativa de la Facultad toda, esa falla será la consecuencia de la ausencia de previsión de esa institución, y no aplicable a todas las revistas. En este caso la existencia o no de esa revista depende solo de esa Unidad Académica, pero en el caso de otras revistas no es así.

Ahora bien, vuelvo a la pregunta inicial porque las respuestas que he dado me siguen pareciendo insatisfactorias. Preguntar de quién es la revista es, de algún modo, lo mismo que preguntar de quién es la 
Facultad o la Unidad Académica, e incluso la Universidad. Voy a completar el interrogante de este modo: ¿de quién es la Facultad sino de todos aquellos que la formamos y sostenemos con nuestro trabajo, apoyo, fomento, empeño, afecto, y nos consideramos parte de ella? Por si no queda claro este concepto, somos parte de la Universidad Pública quienes la hacemos cotidianamente, sin distinción de clase, sexo, religión, tendencia política, nacionalidad, etnia, y todos los etcéteras que se nos ocurran. Es posible que esta posición moleste a un determinado grupo minoritario, o a varios, que piensen que son propietarios de un Instituto, Centro de Investigación, Facultad o Universidad. Considero que se equivocan. Tuve la oportunidad de decirlo hace unos años y quienes suponían lo contrario eludieron la discusión. Lo ratifico: la dinámica universitaria no permite que sea de alguien, o de un grupo o sector. Puede aparentar serlo, solo de forma transitoria. Tarde o temprano las cosas cambian y quienes hoy piensen que pueden reclamar que se haga lo que ellos pretenden, mañana pueden encontrarse en la posición de argumentar lo que vengo sosteniendo para poder sobrevivir en el medio. Siento que ya voy respondiendo mejor. Por la misma razón de que tanto la Universidad, la Facultad, como la revista X no son propiedad de alguien en particular - fuera de la propiedad intelectual, etc., que no viene al caso argumentar - porque son el producto de una actividad colectiva, los artículos que se publican también terminan siendo de todos. Me explico mejor: el contenido de cada uno de los artículos, por el principio que guía y sustenta el modelo de acceso abierto, es de todos. La ciencia no es propiedad de nadie; el conocimiento que ponemos en circulación, tampoco. Solo hay un conjunto de seres humanos que interactuando producen, en forma creativa, una serie de saberes que desean compartir con otros. Entonces, llegado a este punto debo señalar que la pregunta inicial resulta irrelevante y no contribuye a clarificar los procesos de producción involucrados en la edición de una revista científica, sino que provoca una contradicción lógica.

Es bastante evidente que en los últimos tiempos se avanzó mucho en la puesta en circulación de las revistas científicas de las universidades públicas y en particular de la UNLP, razón por la cual se reconoce el trabajo de la gestión editorial, como también el apoyo que las autoridades pudieron dar para lograr la visibilidad alcanzada. Ciertamente las autoridades saben que el conocimiento científico bien divulgado constituye una parte fundamental de la vida misma de la Universidad, logra el reconocimiento entre sus pares y prestigia a la institución. La consecuencia ha sido el apoyo brindado a quienes conocen los aspectos técnicos de la divulgación científica, las indizaciones y los repositorios donde ubicar a nuestras revistas, y se ocuparon de poner orden dentro de un caos de criterios tal vez ya obsoletos. En estas materias me parece advertir un avance en la universalización de las normas que de alguna forma ordenaron el trabajo interno de los equipos editoriales. $^{9}$

El buen trabajo realizado tanto por autoridades como por quienes hacen la gestión editorial ha sido fructífero no solo por sus virtudes, sino - y fundamentalmente- porque encontraron el material disponible para llevar adelante la tarea de difusión. Porque si no hubiera revistas que cumplieran con todos los requisitos que se les piden a los editores respecto de la aplicación de sus criterios de calidad, originalidad y normatividad, no tendrían qué difundir. En otras palabras: existía un producto que podía mejorarse en varios aspectos, pero sobre el cual se podía trabajar para mostrarlo mejor. En este sentido cobran importancia los equipos editoriales, sobre quienes recae el peso de sostener no solo al medio de que se trate, sino de cumplir con las exigencias de los organismos de evaluación. ${ }^{10}$

Pero aún me queda en el tintero (¿qué habrá sido de la pluma y el plumín!) la pregunta sobre lo que debe publicarse. Porque esta también es una pregunta que aparece una y otra vez. Entonces, ¿qué es lo que debe publicarse? Tenemos que volver a las mismas cosas dichas, a saber: se publican los artículos, los dossiers, los comentarios, los debates, las reseñas, las entrevistas, los avances de equipos, los informes científicos, etc., que cada equipo editorial se ha fijado como producto de su política editorial; que - repito- se establece en los alcances y objetivos, o en las metas y los propósitos. En otras palabras, en el ideal que se propusieron alcanzar los grupos o las instituciones que definieron la constitución de la revista. ¿Alcanza con esta respuesta? ¿Cuál es, si no, la respuesta a aquello de qué hay que publicar y qué no? ¿Qué más que las evaluaciones doble 
ciego con todas las exigencias del caso, y que la adecuación a las normativas establecidas? Los editores y el "colegio invisible" de especialistas son los responsables de determinar qué artículo reúne las condiciones para ser publicado, respetando los dos parámetros que se establecen como fundamentales para la evaluación, a saber: la revisión por pares de los documentos editados y la exigencia de originalidad (Aparicio, Banzato y Liberatore, 2016).

En síntesis, las revistas son espacios creados por diferentes actores que expresan distintas tendencias, enfoques disciplinarios, voluntades políticas, etc., todas transitorias. Queda a juicio (igualmente transitorio) de las unidades académicas, o de la propia Universidad — a través de sus órganos colectivos representativossugerir y no reclamar o imponer criterios a contramano de las prácticas de inclusión y diversidad, precisamente en un ámbito donde debe fomentarse la crítica (incluso al propio espacio) porque de ello depende el futuro de la Universidad Pública. Los equipos editoriales estamos tensionados por los aspectos lógicos de las tareas internas de cualquier órgano de divulgación, pero en los últimos años se han sumado otros actores que también tensionan la actividad. Esto se da en gran medida porque las revistas desean adecuarse a las pautas nacionales e internacionales que, muchas veces, entran en contradicción con lo que venía haciéndose. En este sentido, sugiero partir del respeto por lo que en cada espacio se hace, dialogando sobre todos los asuntos que se quieran establecer, modificar, o sobre los nuevos parámetros que se pretenda implementar. Porque cada uno de esos espacios - las autoridades, los equipos de gestión editorial y los editores - conocen sus tareas específicas y por ninguna razón estas deben confundirse.

\section{ReFERENCiAS}

Abadal, E., y Alcaraz, L. R. (2006). Revistas científicas digitales: características e indicadores. RUSC. Universities and Knowledge Society Journal, 3(1), 20. http://dx.doi.org/10.7238/rusc.v3i1.272

Alperin, J. P. y Rozemblum, C. (2017). La reinterpretación de visibilidad y calidad en las nuevas políticas de evaluación de revistas científicas. Revista Interamericana de Bibliotecología, 40(3), 231-241. https://doi.org/10.17533/ude a.rib.v40n3a04

Altamirano, C. (2006). Intelectuales. Notas de investigación. Bogotá: Norma.

Altamirano, C. (dir.) (2008). Historia de los intelectuales en América Latina (vol I-II). Buenos Aires: Katz Editores.

Aparicio, A., Banzato, G., y Liberatore, G. (2016). Manual de gestión editorial de revistas cientificas de ciencias sociales $y$ humanas: Buenas prácticas y criterios de calidad. Ciudad Autónoma de Buenos Aires: CLACSO: CAICYTCONICET: PISAC: MINCyT: REUN. Recuperado de http://www.memoria.fahce.unlp.edu.ar/libros/pm.48 2/pm.482.pdf

Barriera, D. (Dir) (2018).Justicias situadas. Entre el Virreinato Rioplatense y la República Argentina (1776-1864). La Plata: Universidad Nacional de La Plata, Facultad de Humanidades y Ciencias de la Educación. Instituto de Investigación en Humanidades y Ciencias Sociales.

Bauman, Z. (2005). Legisladores e intérpretes. Sobre la modernidad, la posmodernidad y los intelectuales. Buenos Aires: Universidad Nacional de Quilmes.

Beigel, F. (2018a). Las relaciones de poder en la ciencia mundial. Un anti-ranking para conocer la ciencia producida en la periferia. Nueva Sociedad, 274, 13-28. Recuperado de https://nuso.org/articulo/las-relaciones-de-poder-e n-la-ciencia-mundial/

Beigel, F. (2018b). Un mundo de circuitos: el desplazamiento desde el impacto a la circulación [mensaje en el blog Ameli]. Recuperado de http://www.amelica.org/index.php/2018/11/27/un-mundo-de-circuitos-el-desplazam iento-desde-el-impacto-a-la-circulacion/

Bobbio, N. (1998). La duda y la elección. Intelectuales y poder en la sociedad contemporánea. Barcelona: Paidós.

Borrego, Á., y Urbano, C. (2006). La evaluación de revistas científicas en Ciencias Sociales y Humanidades. Información, cultura y sociedad, 14, 11-27. Recuperado de http://revistascientificas.filo.uba.ar/index.php/ICS/ article/view/886 
Bourdieu, P. (2005). Intelectuales, política y poder. Buenos Aires: Eudeba.

Casal, G.B. (2003). Evaluación de la calidad de los artículos y de las revistas científicas: Propuesta del factor de impacto ponderado y de un índice de calidad. Psicothema, 15(1), 23-35. Recuperado de http://www.psicothema.com/ pdf/400.pdf

Devés Valdés, E. (2000). Redes intelectuales en América Latina. Santiago de Chile: Instituto de Estudios Avanzados, Universidad Santiago de Chile.

Hofmeister, W. y H.C.F. Mansilla (eds.) (2003). Intelectuales y politica en América Latina. Rosario: Homo Sapiens.

Mendoza, S. y Paravic, T. (2006). Origen, clasificación y desafíos de las revistas científicas. Investigación y postgrado, 21(1), 49-75. Recuperado de http://www.scielo.org.ve/scielo.php?pid=S1316-00872006000100003\&script=s ci_arttext

Monsiváis, C. (2007). De los intelectuales en América Latina. América Latina Hoy, 47, 15-38. Recuperado de http:/ /revistas.usal.es/index.php/1130-2887/article/view/1365

Ramírez Martínez, D. C., Martínez Ruiz, L. C. y Castellanos Domínguez, Ó. F. (2012). Divulgación y difusión del conocimiento: las revistas cientificas. Universidad Nacional de Colombia. Recuperado de http://bdigital.unal.ed u.co/8394/1/9789587613346.pdf

Rozemblum, C. y Banzato, G. (2012). La cooperación entre editores y bibliotecarios como estrategia institucional para la gestión de revistas científicas. Información, cultura y sociedad, 27, 91-106. Recuperado de http://revistascient ificas.filo.uba.ar/index.php/ICS/article/view/686

Rozemblum, C., Unzurrunzaga, C., Banzato, G. y Pucacco, C. (2015). Calidad editorial y calidad científica en los parámetros para inclusión de revistas científicas en bases de datos en Acceso Abierto y comerciales. Palabra Clave (La Plata), 4(2), 64-80. Recuperado de https://www.palabraclave.fahce.unlp.edu.ar/article/view/PCv4n2a01/

Rozemblum, C., Unzurrunzaga, C., Pucacco, C., y Banzato, G. (2013). Parámetros de evaluación para la inclusión e indización de revistas científicas en bases de datos locales e internacionales. En Actas de las VII Jornadas de Sociologia de la Universidad Nacional de La Plata. Argentina en el escenario latinoamericano actual: debates desde las ciencias sociales. La Plata. Recuperado de http://sedici.unlp.edu.ar/handle/10915/31377

Rozemblum, C., y Banzato, G. (2009). Nuevos roles del bibliotecario en las estrategias de publicación de revistas académicas. En VII Encuentro de Editores de Revistas Cientificas. La Habana, Cuba. Consejo Editorial de la Revista Cubana de Ciencia Agrícola. Recuperado de http://www.memoria.fahce.unlp.edu.ar/trab_eventos/ev. 522/ev.522.pdf

Said, E. W. (1996). Las representaciones del intelectual. España: Paidós.

Williams, R. (1976). Keywords a vocabulary of culture and society. New York: Oxford University Press.

\section{Notas}

1 Sin ser exhaustivo, una bibliografía sobre intelectual/es en: Altamirano, 2006 y 2008; Bauman, 2005; Bobbio, 1998 ; Bourdieu, 2005; Devés Valdés, 2000; Hofmeister y Mansilla, 2003; Monsiváis, 2007; Said, 1996; Williams, 1976.

2 Trabajos y Comunicaciones es la revista del Departamento de Historia de la Facultad de Humanidades y Ciencias de la Educación, donde trabajo desde el año 2005. Cuadernos de H Ideas está alojada en la Facultad de Periodismo y Comunicación Social y formo parte de la misma desde su primer número del año 2007.

3 Me referiré siempre a revistas de divulgación en ciencias humanas y sociales, pues desconozco la situación en otros campos científicos.

4 Consejo Nacional de Investigaciones Científicas y Técnicas (https://www.conicet.gov.ar/)

5 Algunas respuestas disponibles y específicas en Ramírez Martínez, Martínez Ruiz y Castellanos Domínguez, 2012; Rozemblum, Unzurrunzaga, Banzato y Pucacco, 2015, 2013; Mendoza y Paravic, 2006.

6 Lo planteado aquí se aplicará siempre a las revistas científicas del área de las ciencias sociales y humanas.

7 También se nos interroga sobre quién fija los criterios para que un artículo sea científico. No eludo la respuesta, solo que aquí abordo otra cuestión. Una visión sobre el tema en Aparicio, Banzato y Liberatore, 2016. También en Beigel, 2018a, 2018b.

8 Algunos de los índices con que se evalúan las revistas en Borrego y Urbano, 2006; Casal, 2003 y Alperin y Rozemblum, 2017. 
9 Considero que las normas a las que nos hemos adecuado tienen muchas virtudes. Aplicadas con un alto grado de rigidez, algunos aspectos dejan de serlo. Dejo este asunto para tratarlo en otro lugar.

10 Una observación sobre el espíritu de colaboración que debe primar entre las partes en Rozemblum y Banzato, 2012. 